\title{
Dynamic stochastic blockmodels: Statistical models for time-evolving networks
}

\author{
Kevin S. $\mathrm{Xu}^{\star}$ and Alfred O. Hero III \\ Department of Electrical Engineering and Computer Science, \\ University of Michigan, Ann Arbor, MI, USA \\ \{xukevin, hero\}@umich.edu
}

\begin{abstract}
Significant efforts have gone into the development of statistical models for analyzing data in the form of networks, such as social networks. Most existing work has focused on modeling static networks, which represent either a single time snapshot or an aggregate view over time. There has been recent interest in statistical modeling of dynamic networks, which are observed at multiple points in time and offer a richer representation of many complex phenomena. In this paper, we propose a state-space model for dynamic networks that extends the well-known stochastic blockmodel for static networks to the dynamic setting. We then propose a procedure to fit the model using a modification of the extended Kalman filter augmented with a local search. We apply the procedure to analyze a dynamic social network of email communication.
\end{abstract}

Keywords: dynamic network, stochastic blockmodel, state-space model

\section{Introduction}

Many complex physical, biological, and social phenomena are naturally represented by networks. Tremendous efforts have been dedicated to analyzing network data, which has led to the development of many formal statistical models for networks. Most research has focused on static networks, which either represent a single time snapshot of the phenomenon being investigated or an aggregate view over time. As such, statistical models for static networks have a long history in statistics and sociology among other fields 2 . However, most complex phenomena, including social behavior, are time-varying, which has led researchers to consider dynamic, time-evolving networks.

In this paper, we consider dynamic networks represented by a sequence of snapshots of the network at discrete time steps. We characterize such networks using a set of unobserved time-varying states from which the observed snapshots are derived. We propose a state-space model for dynamic networks that combines two types of statistical models: a static model for the individual snapshots and a temporal model for the evolution of the states. The network snapshots are modeled using the stochastic blockmodel [5], a simple parametric model commonly

\footnotetext{
^ Current affiliation: 3M Corporate Research Laboratory, St. Paul, MN, USA
} 
used in the analysis of static social networks. The state evolution is modeled by a stochastic dynamic system. Using a Central Limit Theorem approximation, we develop a near-optimal procedure for fitting the proposed model in the on-line setting where only past and present network snapshots are available. The inference procedure involves a modification of the extended Kalman filter, which is used for state tracking in many applications [3], augmented with a local search strategy. We apply the proposed procedure to analyze a dynamic social network of email communication and predict future email activity.

\section{Related work}

Several statistical models for dynamic networks have previously been proposed by extending a static model to the dynamic setting in a similar fashion to our proposed model 22. Two such models include temporal extensions of the exponential random graph model [1] and latent space model [13]. More closely related to the state-space model we propose are several temporal extensions of stochastic blockmodels (SBMs). SBMs divide nodes in the network into multiple classes and generate edges independently with probabilities $\theta_{a b}$ dependent on the class memberships $a, b$ of the nodes 5 . Yang et al. 15 propose a dynamic SBM involving a transition matrix that specifies the probability that a node in class $i$ at time $t$ switches to class $j$ at time $t+1$ for all $i, j, t$ and fit the model using Gibbs sampling and simulated annealing. Ho et al. 4 propose a temporal extension of a mixed-membership version of the SBM using linear state-space models for the class membership vectors of node clusters. One major difference between [4, 15] and this paper is that we treat the edge probabilities $\theta_{a b}$ as time-varying states, while [4, 15] treat them as time-invariant parameters. In addition, our model allows for a simpler inference procedure using a Central Limit Theorem approximation. We demonstrate the importance of the time-varying states for analysis of a dynamic social network in Section 5

\section{Static stochastic blockmodels}

We first introduce notation and summarize the static stochastic blockmodel (SSBM), which we use as the static model for the individual network snapshots. We represent a dynamic network by a time-indexed sequence of graphs, with $W^{t}=\left[w_{i j}^{t}\right]$ denoting the adjacency matrix of the graph observed at time step $t . w_{i j}^{t}=1$ if there is an edge from node $i$ to node $j$ at time $t$, and $w_{i j}^{t}=0$ otherwise. We assume that the graphs are directed, i.e. $w_{i j}^{t} \neq w_{j i}^{t}$ in general, and that there are no self-edges, i.e. $w_{i i}^{t}=0 . W^{(s)}$ denotes the set of all snapshots up to time $s,\left\{W^{s}, W^{s-1}, \ldots, W^{1}\right\}$. The notation $i \in a$ indicates that node $i$ is a member of class $a .|a|$ denotes the number of nodes in class $a$. The classes of all nodes at time $t$ is given by a vector $\boldsymbol{c}^{t}$ with $c_{i}^{t}=a$ if $i \in a$ at time $t$. We denote the submatrix of $W^{t}$ corresponding to the relations between nodes in class $a$ and class $b$ by $W_{[a][b]}^{t}$. We denote the vectorized equivalent of a matrix $X$, 
i.e. the vector obtained by simply stacking columns of $X$ on top of one another, by $\boldsymbol{x}$. Doubly-indexed subscripts such as $x_{i j}$ denote entries of matrix $X$, while singly-indexed subscripts such as $x_{i}$ denote entries of the vectorized equivalent $\boldsymbol{x}$.

Consider a snapshot at an arbitrary time step $t$. An SSBM is parameterized by a $k \times k$ matrix $\Theta^{t}=\left[\theta_{a b}^{t}\right]$, where $\theta_{a b}^{t}$ denotes the probability of forming an edge between a node in class $a$ and a node in class $b$, and $k$ denotes the number of classes. The SSBM decomposes the adjacency matrix into $k^{2}$ blocks, where each block is associated with relations between nodes in two classes $a$ and $b$. Each block corresponds to a submatrix $W_{[a][b]}^{t}$ of the adjacency matrix $W^{t}$. Thus, given the class membership vector $\boldsymbol{c}^{t}$, each entry of $W^{t}$ is an independent realization of a Bernoulli random variable with a block-dependent parameter; that is, $w_{i j}^{t} \sim \operatorname{Bernoulli}\left(\theta_{c_{i}^{t} c_{j}^{t}}^{t}\right)$.

SBMs are used in two settings:

1. The a priori blockmodeling setting, where class memberships are known or assumed, and the objective is to estimate the matrix of edge probabilities $\Theta^{t}$.

2. The a posteriori blockmodeling setting, where the objective is to simultaneously estimate $\Theta^{t}$ and the class membership vector $\boldsymbol{c}^{t}$.

Since each entry of $W^{t}$ is independent, the likelihood for the SBM is given by

$$
\begin{aligned}
f\left(W^{t} ; \Phi^{t}\right) & =\prod_{i \neq j}\left(\theta_{c_{i} c_{j}}^{t}\right)^{w_{i j}^{t}}\left(1-\theta_{c_{i} c_{j}}^{t}\right)^{1-w_{i j}^{t}} \\
& =\exp \left\{\sum_{a=1}^{k} \sum_{b=1}^{k}\left[m_{a b}^{t} \log \left(\theta_{a b}^{t}\right)+\left(n_{a b}^{t}-m_{a b}^{t}\right) \log \left(1-\theta_{a b}^{t}\right)\right]\right\},
\end{aligned}
$$

where $m_{a b}^{t}=\sum_{i \in a} \sum_{j \in b} w_{i j}^{t}$ denotes the number of observed edges in block $(a, b)$, and

$$
n_{a b}^{t}= \begin{cases}|a||b| & a \neq b \\ |a|(|a|-1) & a=b\end{cases}
$$

denotes the number of possible edges in block $(a, b)[6]$. The parameters are given by $\Phi^{t}=\Theta^{t}$ in the a priori setting, and $\Phi^{t}=\left\{\Theta^{t}, \boldsymbol{c}^{t}\right\}$ in the a posteriori setting. In the a priori setting, a sufficient statistic for estimating $\Theta^{t}$ is the matrix $Y^{t}$ of block densities (ratio of observed edges to possible edges within a block) with entries $y_{a b}^{t}=m_{a b}^{t} / n_{a b}^{t}$. $Y^{t}$ also happens to be the maximum-likelihood estimate of $\Theta^{t}$, which can be shown [6] by setting the derivative of the logarithm of (1) to 0 .

Estimation in the a posteriori setting is more involved, and many methods have been proposed, including Gibbs sampling [8, label-switching [6, 16, and spectral clustering [12. The label-switching methods use a heuristic for solving the combinatorial optimization problem of maximizing the likelihood (1) over the set of possible class memberships, which is too large to perform an exhaustive search. 


\section{Dynamic stochastic blockmodels}

We propose a state-space model for dynamic networks that consists of a temporal extension of the static stochastic blockmodel. First we present the model and inference procedure for a priori blockmodeling, and then we discuss the additional steps necessary for a posteriori blockmodeling. The inference procedure is on-line, i.e. the state estimate at time $t$ is formed using only observations from time $t$ and earlier.

\subsection{A priori blockmodels}

In the a priori SSBM setting, $Y^{t}$ is a sufficient statistic for estimating $\Theta^{t}$ as discussed in Section 3 . Thus in the a priori dynamic SBM setting, we can equivalently treat $Y^{t}$ as the observation rather than $W^{t}$. The entries of $W_{[a][b]}^{t}$ are independent and identically distributed (iid) Bernoulli $\left(\theta_{a b}^{t}\right)$; thus by the Central Limit Theorem, the sample mean $y_{a b}^{t}$ is approximately Gaussian with mean $\theta_{a b}^{t}$ and variance $\left(\sigma_{a b}^{t}\right)^{2}=\theta_{a b}^{t}\left(1-\theta_{a b}^{t}\right) / n_{a b}^{t}$, where $n_{a b}^{t}$ was defined in (2). We assume that $y_{a b}^{t}$ is indeed Gaussian for all $(a, b)$ and posit the linear observation model

$$
Y^{t}=\Theta^{t}+Z^{t}
$$

where $Z^{t}$ is a zero-mean iid Gaussian noise matrix with variance $\left(\sigma_{a b}^{t}\right)^{2}$ for the $(a, b)$ th entry.

In the dynamic setting where past snapshots are available, the observations would be given by the set $Y^{(t)}$. The set $\Theta^{(t)}$ can then be viewed as states of a dynamic system that is generating the noisy observation sequence. We complete the model by specifying a model for the state evolution over time. Since $\theta_{a b}^{t}$ is a probability and must be bounded between 0 and 1 , we instead work with the matrix $\Psi^{t}=\left[\psi_{a b}^{t}\right]$ where $\psi_{a b}^{t}=\log \left(\theta_{a b}^{t}\right)-\log \left(1-\theta_{a b}^{t}\right)$, the logit of $\theta_{a b}^{t}$. A simple model for the state evolution is the random walk

$$
\boldsymbol{\psi}^{t}=\boldsymbol{\psi}^{t-1}+\boldsymbol{v}^{t}
$$

where $\boldsymbol{\psi}^{t}$ is the vector representation of the matrix $\Psi^{t}$, and $\boldsymbol{v}^{t}$ is a random vector of zero-mean Gaussian entries, commonly referred to as process noise, with covariance matrix $\Gamma^{t}$. The entries of the process noise vector are not necessarily independent or identically distributed (unlike the entries of $Z^{t}$ ) to allow for states to evolve in a correlated manner. The observation model can then be written in terms of $\boldsymbol{\psi}^{t}$ as ${ }^{1}$

$$
\boldsymbol{y}^{t}=h\left(\boldsymbol{\psi}^{t}\right)+\boldsymbol{z}^{t}
$$

where the function $h: \mathbb{R}^{k^{2}} \rightarrow \mathbb{R}^{k^{2}}$ is defined by $h_{i}(\boldsymbol{x})=1 /\left(1+e^{-x_{i}}\right)$, i.e. the logistic function applied to each entry of $\boldsymbol{x}$. We denote the covariance matrix of $\boldsymbol{z}^{t}$ by $\Sigma^{t}$, which is a diagonal matrix ${ }^{2}$ with entries given by $\left(\sigma_{a b}^{t}\right)^{2}$. A graphical representation of the proposed model for the dynamic network is shown in Fig. 1 .

\footnotetext{
${ }^{1}$ Note that we have converted the block densities $Y^{t}$ and observation noise $Z^{t}$ to their respective vector representations $\boldsymbol{y}^{t}$ and $\boldsymbol{z}^{t}$.

${ }^{2}$ The indices $(a, b)$ for $\left(\sigma_{a b}^{t}\right)^{2}$ are converted into a single index $i$ corresponding to the vector representation $\boldsymbol{z}^{t}$.
} 


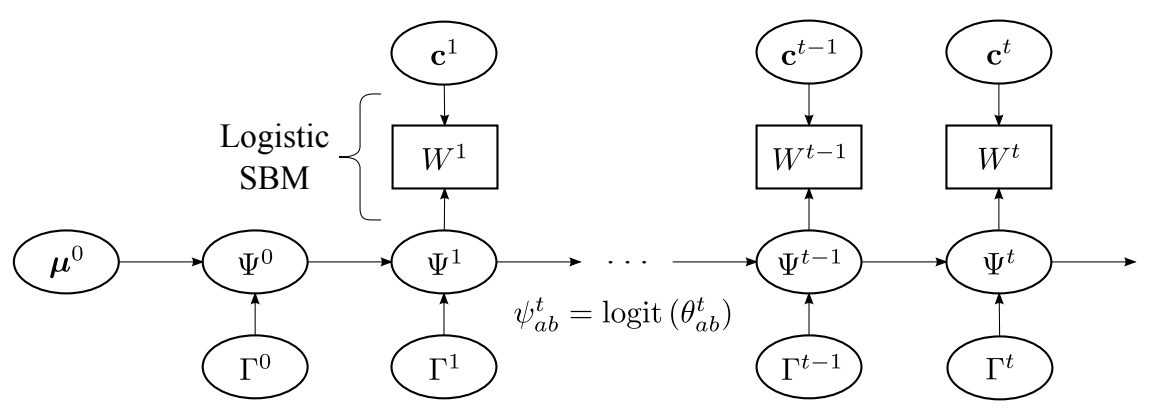

Fig. 1. Graphical representation of the proposed model. The rectangular boxes denote observed quantities, and the ovals denote unobserved quantities. The logistic SBM refers to applying the logistic function to each entry of $\Psi^{t}$ to obtain $\Theta^{t}$ then generating $W^{t}$ using $\Theta^{t}$ and $\boldsymbol{c}^{t}$.

To perform inference on this model, we assume the initial state is Gaussian distributed, i.e. $\boldsymbol{\psi}^{0} \sim \mathcal{N}\left(\boldsymbol{\mu}^{0}, \Gamma^{0}\right)$, and that $\left\{\boldsymbol{\psi}^{0}, \boldsymbol{v}^{1}, \ldots, \boldsymbol{v}^{t}, \boldsymbol{z}^{1}, \ldots, \boldsymbol{z}^{t}\right\}$ are mutually independent. If (3) was linear in $\boldsymbol{\psi}^{t}$, then the optimal estimate of $\boldsymbol{\psi}^{t}$ in terms of minimum mean-squared error would be given by the Kalman filter [3]. Due to the non-linearity, we apply the extended Kalman filter (EKF), which linearizes the dynamics about the predicted state and provides an near-optimal estimate of $\boldsymbol{\psi}^{t}$. The predicted state under the random walk model is simply $\hat{\boldsymbol{\psi}}^{t \mid t-1}=\hat{\boldsymbol{\psi}}^{t-1 \mid t-1}$ with covariance $R^{t \mid t-1}=R^{t-1 \mid t-1}+\Gamma^{t}$. Let $J^{t}$ denote the Jacobian of $h$ evaluated at the predicted state $\hat{\boldsymbol{\psi}}^{t \mid t-1}$. The EKF update equations are as follows [3]:

$$
\begin{array}{lll}
\text { Near-optimal Kalman gain: } & K^{t}=R^{t \mid t-1}\left(J^{t}\right)^{T}\left[J^{t} R^{t \mid t-1}\left(J^{t}\right)^{T}+\Sigma^{t}\right]^{-1} \\
\text { Posterior state estimate: } & \hat{\boldsymbol{\psi}}^{t \mid t}=\hat{\boldsymbol{\psi}}^{t \mid t-1}+K^{t}\left[\boldsymbol{y}^{t}-h\left(\hat{\boldsymbol{\psi}}^{t \mid t-1}\right)\right] \\
\text { Posterior estimate covariance: } & R^{t \mid t}=\left(I-K^{t} J^{t}\right) R^{t \mid t-1}
\end{array}
$$

The posterior state estimate $\hat{\boldsymbol{\psi}}^{t \mid t}$ provides a near-optimal fit to the model at time $t$ given the observed sequence $W^{(t)}$. How to choose the hyperparameters $\left(\boldsymbol{\mu}^{0}, \Gamma^{0}, \Sigma^{t}, \Gamma^{t}\right)$ in an optimal manner is beyond the scope of this paper and is discussed in [14, chap. 5].

\subsection{A posteriori blockmodels}

In many applications, the class memberships $\boldsymbol{c}^{t}$ are not known a priori and must be estimated along with $\Psi^{t}$. This can be done using label-switching methods 6, 16, but rather than maximizing the likelihood, we maximize the posterior state density given the entire sequence of observations $W^{(t)}$ up to time $t$ to account for the prior information. This is done by alternating between labelswitching and applying the EKF. 
The posterior state density is given by

$$
f\left(\boldsymbol{\psi}^{t} \mid W^{(t)}\right) \propto f\left(W^{t} \mid \boldsymbol{\psi}^{t}, W^{(t-1)}\right) f\left(\boldsymbol{\psi}^{t} \mid W^{(t-1)}\right) .
$$

By the conditional independence of current and past observations given the current state, $W^{(t-1)}$ drops out of the first term in (4). It can thus be obtained simply by substituting $h\left(\boldsymbol{\psi}^{t}\right)$ for $\boldsymbol{\theta}^{t}$ in (1). The second term in (4) is equivalent to $f\left(\boldsymbol{\psi}^{t} \mid \boldsymbol{y}^{(t-1)}\right)$ because the class memberships at all previous time steps have already been estimated. By applying the Kalman filter to the linearized temporal model [3], $f\left(\boldsymbol{\psi}^{t} \mid \boldsymbol{y}^{(t-1)}\right) \sim \mathcal{N}\left(\hat{\boldsymbol{\psi}}^{t \mid t-1}, R^{t \mid t-1}\right)$. Thus the logarithm of the posterior density is given by

$$
\begin{aligned}
& \log f\left(\boldsymbol{\psi}^{t} \mid\right.\left.W^{(t)}\right)=c-\frac{1}{2}\left(\boldsymbol{\psi}^{t}-\hat{\boldsymbol{\psi}}^{t \mid t-1}\right)^{T}\left(R^{t \mid t-1}\right)^{-1}\left(\boldsymbol{\psi}^{t}-\hat{\boldsymbol{\psi}}^{t \mid t-1}\right) \\
&+\sum_{a=1}^{k} \sum_{b=1}^{k}\left\{m_{a b}^{t} \log \left[h\left(\psi_{a b}^{t}\right)\right]+\left(n_{a b}^{t}-m_{a b}^{t}\right) \log \left[1-h\left(\psi_{a b}^{t}\right)\right]\right\}
\end{aligned}
$$

where $c$ is a constant term independent of $\boldsymbol{\psi}^{t}$ that can be ignored ${ }^{3}$

We use the log-posterior (5) as the objective function for label-switching. We find that a simple local search (hill climbing) algorithm 11 initialized using the estimated class memberships at the previous time step suffices, because only a small fraction of nodes change classes between time steps in most applications. At the initial time step, we employ the spectral clustering algorithm of Sussman et al. 12] for the SSBM as the initialization.

\section{Application to Enron email network}

We demonstrate the proposed procedure on a dynamic social network constructed from the Enron corpus 9, 10, which consists of about 0.5 million email messages between 184 Enron employees from 1998 to 2002. We place directed edges between employees $i$ and $j$ at time $t$ if $i$ sends at least one email to $j$ during week $t$. Each time step corresponds to a 1-week interval. We make no distinction between emails sent "to", "cc", or "bcc". In addition to the email data, the roles of most of the employees within the company (e.g. CEO, president, manager, etc.) are available, which we use as classes for a priori blockmodeling. Employees with unknown roles are placed in an "others" class.

\subsection{State tracking}

We begin by examining the temporal variation of the states, which we refer to as state tracking. Recall that the states $\Psi^{t}$ correspond to the logit of the edge probabilities $\Theta^{t}$. We first apply the a priori EKF to obtain the state estimates $\hat{\boldsymbol{\psi}}^{t \mid t}$ and their variances (the diagonal of $R^{t \mid t}$ ). Applying the logistic function, we can then obtain the estimated edge probabilities $\hat{\Theta}^{t \mid t}$ with confidence intervals.

\footnotetext{
${ }^{3}$ At the initial time step, $\hat{\boldsymbol{\psi}}^{1 \mid 0}=\boldsymbol{\mu}^{0}$ and $R^{1 \mid 0}=\Gamma^{0}+\Gamma^{1}$.
} 


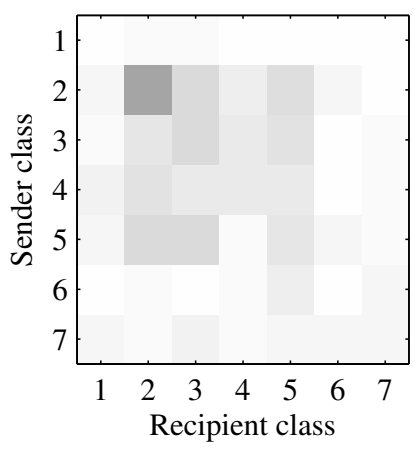

(a) Week 59: a normal week

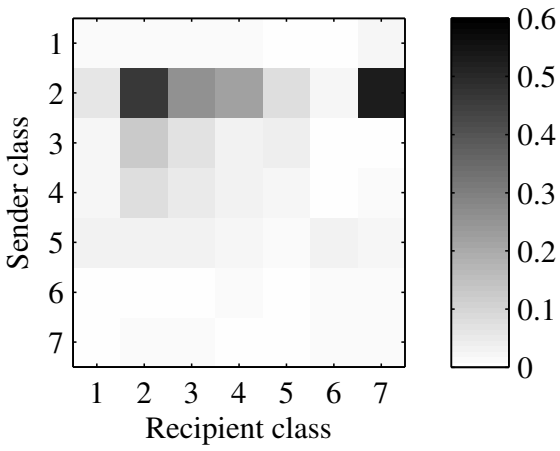

(b) Week 89: CEO Skilling resigns

Fig. 2. Estimated edge probability matrices for two selected weeks. Entry $(i, j)$ denotes the estimated probability of an edge from class $i$ to class $j$. Classes are as follows: (1) directors, (2) CEOs, (3) presidents, (4) vice-presidents, (5) managers, (6) traders, and (7) others. Notice the increase in the probability of edges from CEOs during the week of Skilling's resignation.

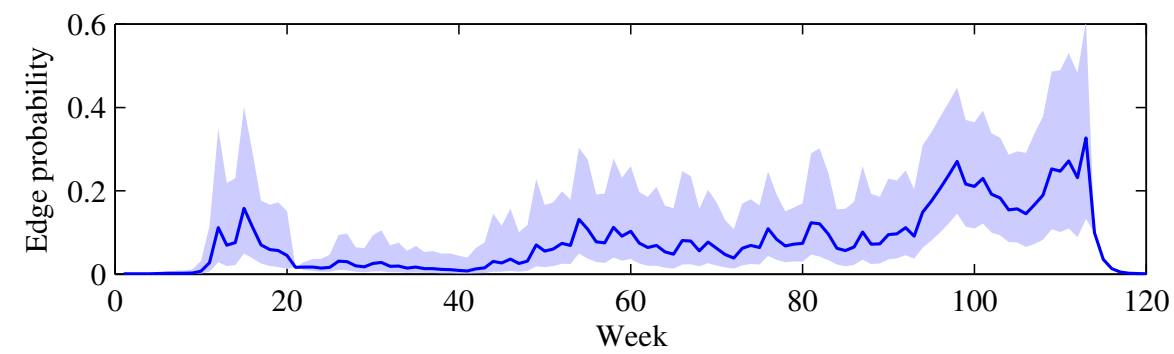

(a) Presidents to presidents

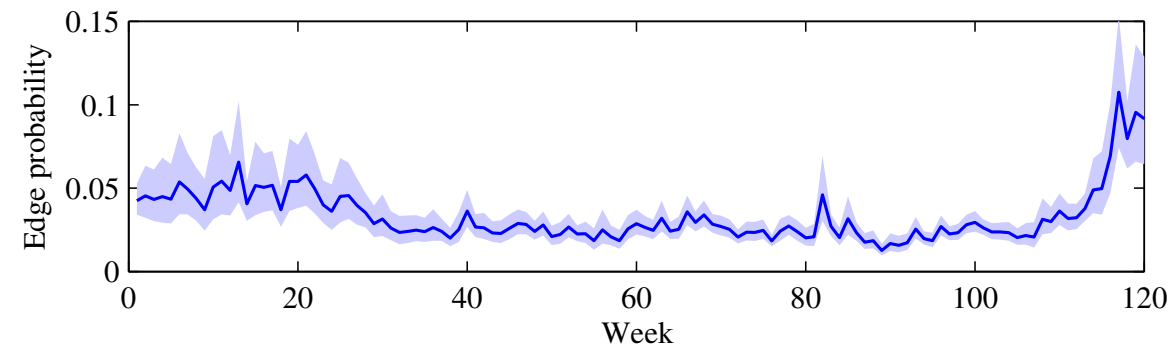

(b) Others to others

Fig. 3. A priori EKF estimated edge probabilities $\hat{\theta}_{a b}^{t \mid t}$ (solid lines) with $95 \%$ confidence intervals (shaded region) for selected $a, b$ by week. An increase in edge probabilities between Enron presidents (a) occurs prior to a similar increase between those in other roles (b) suggesting insider knowledge. 
Examining the temporal variation of $\hat{\Theta}^{t \mid t}$ reveals some interesting trends. For example, a large increase in the probabilities of edges from CEOs is found at week 89. This is the week in which CEO Jeffrey Skilling resigned and is confirmed to be the cause of the increased probabilities by examining the content of the emails. Fig. 2 shows a comparison of the matrix $\hat{\Theta}^{t \mid t}$ during a normal week and during the week Skilling resigned.

Another interesting trend is highlighted in Fig. 3, where the temporal variation of two selected edge probabilities over the entire data trace with $95 \%$ confidence intervals is shown. Edge probabilities between Enron presidents show a steady increase as Enron's financial situation worsens, hinting at more frequent and widespread insider discussions, while emails between others (not of one of the six known roles) begin to increase only after Enron falls under federal investigation.

A key observation from this analysis is the importance of modeling the edge probabilities as time-varying states, as opposed to time-invariant parameters as in [4, 15. Indeed the temporal variation of the edge probabilities is what reveals the internal dynamics of this time-evolving social network. Furthermore, the temporal model provides estimates with less uncertainty than the static SBM, with $95 \%$ confidence intervals that are $24 \%$ narrower on average.

\subsection{Dynamic link prediction}

Next we turn to the task of dynamic link prediction, which differs from static link prediction [7] because the link predictor must simultaneously predict the new edges that will be formed at time $t+1$, as well as the current edges (as of time $t$ ) that will disappear at time $t+1$, from the observations $W^{(t)}$. The latter task is not addressed by most static link prediction methods in the literature.

Since the SBM assumes stochastic equivalence between nodes in the same class, the EKF alone is only a good predictor of the block densities $Y^{t}$, not the edges themselves. However, the EKF can be combined with a predictor that operates on individual edges to form a link predictor. A simple individuallevel predictor is the exponentially-weighted moving average (EWMA) given by $\hat{W}^{t+1}=\lambda \hat{W}^{t}+(1-\lambda) W^{t}$. Using a convex combination of the EKF and EWMA predictors, we obtain a better link predictor that incorporates both block-level characteristics (through the EKF) and individual-level characteristics (through the EWMA). This can be seen from the receiver operating characteristic (ROC) curves in Fig. 4. The a posteriori EKF slightly outperforms the a priori EKF because the a posteriori EKF finds a better fit to the dynamic SBM via a better assignment of nodes to classes than the a priori (assumed) assignment.

\section{Conclusion}

This paper proposes a statistical model for dynamic networks that utilizes a set of unobserved time-varying states to characterize the dynamics of the network. The proposed model extends the well-known stochastic blockmodel for static 


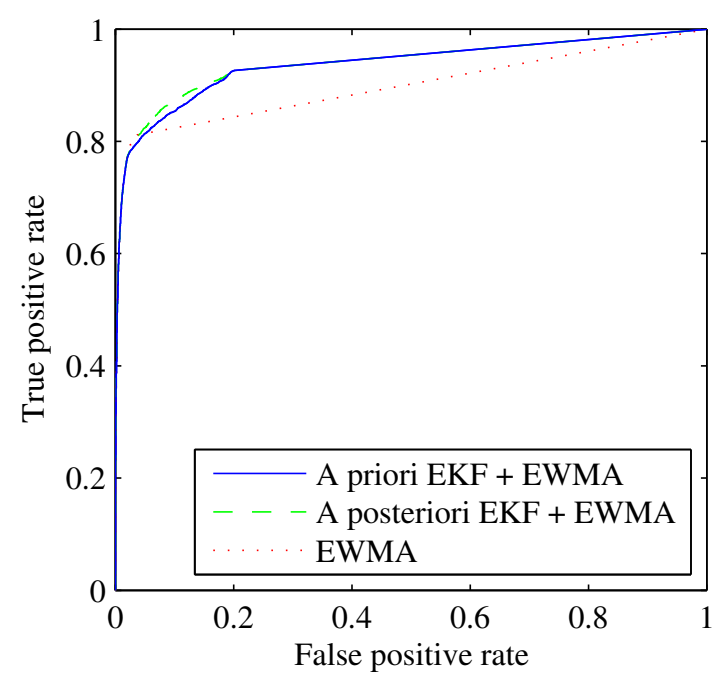

Fig. 4. Comparison of ROC curves for link prediction on Enron data. True positive rate denotes the fraction of actual edges that are correctly predicted, and false positive rate denotes the fraction of non-edges that are predicted to be edges. The convex combination of either EKF with the EWMA outperforms the EWMA alone by accounting for block-level characteristics.

networks to the dynamic setting can be used for either a priori or a posteriori blockmodeling. The main contribution of the paper is a near-optimal on-line inference procedure for the proposed model using a modification of the extended Kalman filter, augmented with a local search. We applied the proposed inference procedure to the Enron email network and discovered some interesting trends when we examined the estimated states. One such trend was a steady increase in emails between Enron presidents as Enron's financial situation worsened, while emails between other employees remained at their baseline levels until Enron fell under federal investigation. In addition, the proposed procedure showed promising results for predicting future email activity. We believe the proposed model and inference procedure can be applied to reveal the internal dynamics of many other dynamic networks.

Acknowledgments. This work was partially supported by the Army Research Office grant W911NF-12-1-0443. Kevin Xu was partially supported by an award from the Natural Sciences and Engineering Research Council of Canada.

\section{References}

[1] Ahmed, A., Xing, E.P.: Recovering time-varying networks of dependencies in social and biological studies. Proc. Nat. Acad. Sci. 106(29), 11878-11883 (2009) 
[2] Goldenberg, A., Zheng, A.X., Fienberg, S.E., Airoldi, E.M.: A survey of statistical network models. Found. Trends Mach. Learn. 2(2), 129-233 (2010)

[3] Haykin, S.: Kalman filtering and neural networks. Wiley-Interscience (2001)

[4] Ho, Q., Song, L., Xing, E.P.: Evolving cluster mixed-membership blockmodel for time-varying networks. In: Proceedings of the 14th Int. Conf. Artif. Intell. Statist. (2011)

[5] Holland, P.W., Laskey, K.B., Leinhardt, S.: Stochastic blockmodels: First steps. Soc. Netw. 5(2), 109-137 (1983)

[6] Karrer, B., Newman, M.E.J.: Stochastic blockmodels and community structure in networks. Phys. Rev. E 83, 016107 (2011)

[7] Liben-Nowell, D., Kleinberg, J.: The link-prediction problem for social networks. J. Am. Soc. Inf. Sci. 58(7), 1019-1031 (2007)

[8] Nowicki, K., Snijders, T.A.B.: Estimation and prediction for stochastic blockstructures. J. Am. Stat. Assoc. 96(455), 1077-1087 (2001)

[9] Priebe, C.E., Conroy, J.M., Marchette, D.J., Park, Y.: Scan statistics on Enron graphs. Comput. Math. Organ. Theory 11(3), 229-247 (2005)

[10] Priebe, C.E., Conroy, J.M., Marchette, D.J., Park, Y.: Scan statistics on Enron graphs (2009), http://cis.jhu.edu/ parky/Enron/enron.html

[11] Russell, S.J., Norvig, P.: Artificial intelligence: A modern approach. Prentice Hall, 2nd edn. (2003)

[12] Sussman, D.L., Tang, M., Fishkind, D.E., Priebe, C.E.: A consistent adjacency spectral embedding for stochastic blockmodel graphs. arXiv:1108.2228v3 [stat.ML] (2012)

[13] Westveld, A.H., Hoff, P.D.: A mixed effects model for longitudinal relational and network data, with applications to international trade and conflict. Ann. Appl. Stat. 5(2A), 843-872 (2011)

[14] $\mathrm{Xu}, \mathrm{K} . \mathrm{S} .:$ Computational methods for learning and inference on dynamic networks. Ph.D. thesis, University of Michigan (2012)

[15] Yang, T., Chi, Y., Zhu, S., Gong, Y., Jin, R.: Detecting communities and their evolutions in dynamic social networks - a Bayesian approach. Mach. Learn. 82(2), 157-189 (2011)

[16] Zhao, Y., Levina, E., Zhu, J.: Consistency of community detection in networks under degree-corrected stochastic block models. The Annals of Statistics (in press) (2012) 